\title{
Sciences environnementales et interdisciplinarité : une réflexion partant des débats aux Pays-Bas
}

\author{
Pieter Leroy \\ Sociologue, PO Box 9108, 6500 HK Nijmegen, Pays-Bas
}

\begin{abstract}
À travers l'exemple des Pays-Bas, c'est en fait une ouverture internationale sur les débats autour de la façon de concevoir la recherche sur les problèmes d'environnement qu'offre cet article. Il est symptomatique que ces débats soient focalisés sur la question de l'interdisciplinarité et que celle-ci soit vue comme une dimension à la fois incontournable et problématique de ces recherches. On ne manquera pas d'être frappé par les similitudes avec la situation française. Signe que l'on se trouve bien là face à des questions de fond. Des questions que ce texte nous met sous les yeux et sur lesquelles il nous invite à réfléchir.
\end{abstract}

La Rédaction

\section{Mots-clés :}

Interdisciplinarité ; sciences de l'environnement; développement durable et défi scientifique ; Pays-Bas et Europe

\section{Keywords:} Interdisciplinarity; environmental sciences; sustainable development and scientific challenge; The Netherlands and Europe

\begin{abstract}
Résumé - Le concept d'interdisciplinarité a occupé une place capitale dans les réflexions sur les sciences de l'environnement dès leur origine, au début des années 1970. Bien que l'interdisciplinarité soit généralement considérée comme une caractéristique de ces sciences, sa mise en œuvre effective, tant dans la recherche que dans l'enseignement, se heurte à des difficultés d'ordre intellectuel et organisationnel. L'auteur commence par exposer l'évolution des façons de la concevoir. Il s'appuie pour cela sur les débats à son propos qui accompagnèrent le développement des sciences de l'environnement aux Pays-Bas ; mais ceux-ci illustrent un mouvement plus général. La référence au développement durable a, à son tour, influencé les discussions sur la question. Dans un second temps est proposée une évaluation des apports de ces débats, des avancées qu'ils ont permises, mais aussi de leurs limites et de leurs échecs. L'article se termine par un examen de la manière dont l'interdisciplinarité, telle qu'elle est conçue par l'auteur, pourrait être organisée tant dans le domaine de l'enseignement que de la recherche.
\end{abstract}

\begin{abstract}
Environmental sciences and interdisciplinarity: a reflection from a Dutch perspective. Right from their start in the early 1970s to the present day, the concept of interdisciplinarity played a major role in the development of environmental sciences, while taking their part in the search for sustainable development. Though interdisciplinarity therefore seems to be a self-evident characteristic and advantage of environmental sciences, its actual implementation, both in research and education, encounters intellectual and organisational difficulties. The first section of this paper highlights the debate on the interdisciplinarity issue. While focusing on the arguments brought forward in three more or less consecutive stages of the history of environmental sciences in The Netherlands, the article sums up arguments and debates that have been held on a much wider scale. The contemporary debate on interdisciplinarity, by the way, is particularly affected by the concept of sustainable development. The second section of the article assesses the benefits and the limits of the interdisciplinarity debate thus far, looking for some critical factors for success and failure. The third section examines the way(s) interdisciplinarity, as we conceive it, could be organised, both in research and education. Although interdisciplinarity is looked upon not so much as an intellectual, but rather as an organisational issue here, we assess some attitudinal characteristics to be necessary for its successful implementation.
\end{abstract}

Auteur correspondant : p.leroy@nsm.kun.nl P. Leroy est professeur de sciences politiques de l'environnement au département de Sciences politiques de l'environnement de la faculté des Sciences de la gestion de l’Université de Nimègue (Pays-Bas). 
Les développements qui suivent sont consacrés à une réflexion sur certains aspects de la question de l'interdisciplinarité dans les sciences environnementales. Le concept d'interdisciplinarité, en France comme ailleurs, a en effet occupé une place capitale dans les sciences de l'environnement dès leur émergence en tant que telles, au début des années 1970. Ce rôle ne s'est jamais démenti et, aujourd'hui, il en va de même dans la recherche en vue d'un développement durable. Bien que l'interdisciplinarité semble caractériser de façon évidente les sciences environnementales dans la définition qu'elles se donnent d'elles-mêmes, sa mise en œuvre effective, tant dans la recherche que dans l'enseignement, se heurte à des difficultés, notamment d'ordre intellectuel et organisationnel.

Dans une première partie, on exposera les termes du débat engagé sur l'interdisciplinarité, à la fois dans l'histoire des sciences environnementales et dans leur évolution la plus récente. Les expériences et les débats qui ont eu lieu aux Pays-Bas en sont une bonne illustration. On s'appuiera donc sur eux, sans pour autant oublier qu'ils renvoient à une échelle plus large, voire à un contexte international. La seconde partie sera consacrée à une évaluation des avancées et des limites de ce débat et à la recherche de critères d'évaluation de sa réussite ou de son échec. Enfin, dans la troisième et dernière partie, sera abordée la question de la façon d'organiser l'interdisciplinarité, tant dans la recherche que dans l'enseignement.

\section{L'interdisciplinarité : un élément essentiel du discours des sciences environnementales}

L'émergence de la question environnementale au début des années 1970 s'est accompagnée du développement d'un nouveau champ de recherches scientifiques, les sciences environnementales. La position de celles-ci (la reconnaissance dont elles jouissent au plan universitaire, leur institutionnalisation, leur importance dans la société, leur impact politique, etc.) varie considérablement d'un pays à l'autre. Loin d'être stabilisé, leur contenu connaît également de grandes différences selon les pays et ceci vaut également pour leurs formes d'organisation. Mais, fait remarquable, en dépit de tous ces facteurs d'hétérogénéité, elles se sont toujours et partout, dès leurs débuts, définies comme interdisciplinaires. Ce n'est pas le lieu de revenir ici longuement sur les définitions. Précisons simplement qu'on se place dans la perspective de sciences sociales ayant un rôle à jouer dans la question environnementale; l'interdisciplinarité dont il est ici question inclut donc à la fois les sciences de la nature et les sciences sociales.
Cette interdisciplinarité s'est trouvée justifiée par des arguments d'ordres différents, tantôt normatifs, tantôt théoriques, tantôt pragmatiques, sur lesquels on reviendra. En bref, ces arguments reviennent à constater que les problèmes environnementaux dépassent les frontières des disciplines scientifiques modernes telles qu'elles se sont développées au XIX ${ }^{\mathrm{e}}$ siècle et au début du XXe . C'est parce qu'il apparaît que l'analyse des problèmes environnementaux et l'élaboration d'une réponse possible à ces problèmes nécessitent des connaissances et des compétences issues de nombreuses disciplines scientifiques que l'interdisciplinarité, définie provisoirement comme l'intégration de connaissances d'une multiplicité de disciplines, apparaît comme étant une condition préalable essentielle aux sciences de l'environnement (La Rivière, 1991 ; O’Riordan, 1995 ; Zanoni, 1997).

Ce point mérite d'être souligné : c'est bien parce qu'il $\mathrm{s}^{\prime}$ agit pour les sciences environnementales de traiter un problème appelant une action, et de le faire dans la perspective de lui trouver une solution, que l'interdisciplinarité est vue comme une nécessité pour elles. De fait, cet objectif suppose que le problème en question soit analysé simultanément dans toutes ses dimensions (ou, au moins, dans les plus importantes d'entre elles). Cette vocation des sciences environnementales à ouvrir des pistes pour la résolution des problèmes, et le besoin d'interdisciplinarité qui en résulte, se reflètent bien dans deux définitions très représentatives de celles qui en sont généralement données. C'est ainsi qu'aux Pays-Bas, elles ont été définies comme «la science interdisciplinaire traitant des relations entre l'homme et son environnement et des problèmes potentiels et effectifs contenus dans ces relations, et devant servir de socle à la résolution ou à la prévention de ces problèmes " (Udo de Haes, 1984). En Angleterre, Nebel et Wright (1993) recourent à une définition analogue lorsqu'ils constatent que la science environnementale « est la science qui étudie les relations de cause à effet qui sous-tendent les questions environnementales », ce qui implique que ces relations dépassent les frontières qui délimitent les disciplines scientifiques.

En d'autres termes, selon ces définitions, le besoin d'interdisciplinarité est en premier lieu inspiré par la recherche d'une pertinence sociétale et politique, et non par les dynamiques inhérentes aux disciplines universitaires elles-mêmes (Hollaender, 2001). Or, le plaidoyer pour l'interdisciplinarité impliquait, du moins initialement, et implique encore dans beaucoup de pays, une démarcation claire entre les sciences environnementales, de développement récent, d'une part, et les disciplines universitaires traditionnelles, d'autre part. C'est pourquoi, bien que l'interdisciplinarité des sciences environnementales ait pu aller de soi pour ceux qui s'y consacraient, il n'en allait pas de même du point de vue des disciplines universitaires établies. 
D'où les nombreuses querelles universitaires sur la question, qui ont accompagné la reconnaissance progressive des sciences environnementales par les universités, les organismes de recherche scientifique et les organes gouvernementaux. Certaines de ces difficultés - on y reviendra - montrent bien que l'interdisciplinarité, comprise comme intégration de connaissances scientifiques à partir de différentes disciplines, n'est pas seulement une question intellectuelle, mais bien plutôt une question organisationnelle.

On présentera les termes du débat sur l'interdisciplinarité de deux manières. Tout d'abord, en revenant sur presque trente années de discussions sur le sujet aux Pays-Bas. En effet, c'est une question qui a joué un grand rôle dans le développement des sciences environnementales dans ce pays, en particulier en ce qui concerne leur différenciation vis-à-vis des disciplines traditionnelles. On s'en tiendra ici aux différentes conceptions de l'interdisciplinarité qui ont eu cours durant cette période et aux concepts mouvants autour desquels elles ont tourné ${ }^{1}$. Dans un deuxième temps, on évoquera la question du rapport entre «durabilité » et interdisciplinarité. La «durabilité » s'est vue considérée, à juste titre, comme un cliché recouvrant un pseudo-consensus. Dans le même temps, néanmoins, ce concept a été largement accepté comme fil conducteur de la politique et de la recherche environnementales. Aussi a-t-il indéniablement suscité de nouvelles discussions sur l'interdisciplinarité, que l'on commentera brièvement.

\section{L'interdisciplinarité dans les sciences environnementales aux Pays-Bas}

L'interdisciplinarité a joué un rôle majeur dans le processus d'institutionnalisation des sciences environnementales aux Pays-Bas. D'où l'étendue et l'importance du débat sur la question dans ce pays (Leroy, 1995; Broekhans, 2003). Bien que, dès le début, l'idée que l'interdisciplinarité était un préalable essentiel aux sciences environnementales y ait été acceptée, de nombreuses discussions ont eu lieu. Ces discussions ont révélé différents points de vue sur l'interdisciplinarité recherchée par les uns et les autres, différentes façons de la concevoir et différentes stratégies pour l'organiser. On peut schématiquement distinguer trois conceptions essentielles, représentant les trois principaux courants sur la question et fondées sur trois arguments différents : l'argument normatif, l'argument scientifique et l'argument pragmatique.

\footnotetext{
1 Pour de plus amples développements, voir Leroy (1995) et Broekhans (2003).
}

\section{L'argument normatif : les sciences environnementales comme contre-courant universitaire}

Les pionniers des sciences environnementales néerlandaises ont principalement justifié l'interdisciplinarité par des arguments normatifs. Pour eux, les problèmes environnementaux semblaient en premier lieu résulter d'un réductionnisme rationaliste, fruit d'une pratique scientifique monodisciplinaire : la spécialisation scientifique impliquant la réduction du champ de chaque discipline, n'y subsiste qu'un petit nombre de variables essentielles, toutes les autres étant considérées comme externes à la discipline. Cette pratique du ceteris paribus des disciplines hautement spécialisées a littéralement conduit à l'externalisation de tous les phénomènes considérés comme négligeables pour telle ou telle discipline particulière. La formule du ceteris paribus, toutes choses étant égales par ailleurs, signifie en réalité cetera desunt: le reste n'existe pas $^{2}$. Ainsi, l'économie classique, par exemple, comme 1'a montré Mishan (1968) déjà à la fin des années 1960, n'accorde aucune attention aux conséquences négatives de la croissance économique et de ses effets. Le recours à la notion d' $\mathrm{d}^{\prime}$ effets externes », caractéristique de cette démarche durant, précisément, les années de la formation de la question environnementale, est particulièrement significatif de ces dérives de la spécialisation scientifique sans cesse croissante, qui impliquait évidemment qu'on néglige ces « effets secondaires » dont les problèmes environnementaux sont l'exemple par excellence.

Bien qu'ils aient clairement rejeté la spécialisation monodisciplinaire et plaidé en faveur de l'interdisciplinarité, ces chercheurs ne présentaient pas d'alternative claire à l'organisation hyper-fragmentée des sciences. Ils n'avaient pas non plus une bonne compréhension de la façon dont les différentes disciplines devaient s'entrecroiser pour forger l'interdisciplinarité. Une idéologie normative et holistique antimoderniste : voilà sans doute comment qualifier au mieux leurs idées, très proches de la vision du monde prédominante dans le mouvement écologiste des débuts (Jamison, 1996). Si la plupart de ces premiers chercheurs en sciences de l'environnement n'avaient pas les ambitions que Roszak (1972) prêtait à l'écologie ( «un art de guérir »), ils suivaient la voie tracée par cette science critique. Avec cette dernière, les sciences environnementales formaient, à leurs débuts, un contre-courant universitaire, prônant l'interdisciplinarité comme caractéristique principale de leur idéologie.

\footnotetext{
${ }^{2} \mathrm{Ou}$, selon le jeu de mots : ceteris paribus, le reste étant égal ; cetera desunt, le reste nous est égal...
} 
L'argument scientifique : la science environnementale comme superdiscipline

Cet argument normatif a progressivement perdu de son poids à mesure que la science environnementale néerlandaise, au début des années 1980, semblait parvenir à ses fins et à s'organiser comme domaine de recherche indépendant. Alors qu'un premier manuel posait les jalons d'un langage commun, les premiers professeurs prenaient leur poste, une revue scientifique voyait le jour et une association professionnelle était créée. La science environnementale néerlandaise s'institutionnalisait rapidement comme domaine universitaire reconnu. Cette évolution institutionnelle s'accompagnait d'un glissement dans les ambitions en matière d'interdisciplinarité et dans les conceptions de cette notion. Certains universitaires souhaitaient que la science environnementale - au singulier ! - devienne une discipline superdisciplinaire. La première édition du Basisboek Milieukunde (Manuel de science environnementale) (1984), par exemple, privilégiait une interdisciplinarité envisagée en termes de contenu, à un niveau conceptuel ou paradigmatique, affirmant que «la » science environnementale se devait de développer un ensemble spécifique de connaissances, ses propres méthodes, etc. Les ambitions de ses auteurs étaient quelque peu excessives, puisque certains chercheurs visaient l'interconnexion de « toutes les connaissances requises pour analyser et résoudre les problèmes environnementaux » au sein d'une seule et même science environnementale (Jórgensen et Johnsen, 1981). Bien qu'il ait été publié presque dix ans plus tard, l'ouvrage intitulé Environmental Science Theory (Théorie de la science environnementale) de De Groot (1992) fait écho à ces ambitions, affirmant que la science environnementale n'est pas seulement la somme de spécialités environnementales monodisciplinaires, mais doit constituer « un pas en avant vers un échelon disciplinaire supérieur disposant de son propre corps de connaissances ». L'interdisciplinarité ne semblait ainsi qu'une étape transitoire sur le chemin d'une science environnementale supradisciplinaire.

Si le courant normatif ne se faisait qu'une idée vague de la façon dont les disciplines pouvaient être mises en relation entre elles pour forger l'interdisciplinarité, la conception scientifique unitaire impliquait, quant à elle, une hiérarchisation claire des disciplines et des champs d'étude scientifique. Dans ce modèle, les disciplines traditionnelles « de base » (de la psychologie au droit ou de la biologie à la toxicologie) étaient censées faire remonter leurs connaissances et compétences pertinentes, par le biais des « spécialités environnementales » (comme la sociologie environnementale, la chimie environnementale, etc.), jusqu'au sommet de la pyramide, la science environnementale en train de naître. Le modèle impliquait un processus de «finalisation par étapes » de la connaissance scientifique, modelée par des concepts interdisciplinaires englobant à la fois les sciences de la nature et les sciences sociales, et aussi bien des faits que des valeurs. Le développement durable, encore peu connu à l'époque - au début des années 1980 - aurait été un bon candidat en la matière.

\section{L'argument pragmatique : l'interdisciplinarité dépendant des problèmes et des opportunités}

Au cours des années 1980, il est devenu évident que ni les programmes d'enseignement des sciences de l'environnement, ni les projets de recherches environnementales - bien qu'ils aient les uns et les autres connu beaucoup de succès dans cette période - ne parvenaient à atteindre le niveau d'interdisciplinarité qui avait été envisagé. Les distances et les différences qui séparaient les disciplines impliquées, en termes d'épistémologies, de concepts, de théories et de méthodes, ainsi qu'en termes de priorité et de pertinence pour les problèmes environnementaux, rendaient plus complexe que prévu le développement de l'interdisciplinarité. La seconde édition du Basisboek Milieukunde (1991) observait que les opinions divergeaient quant aux chances de parvenir à intégrer les différents cadres conceptuels, théories et méthodes des spécialités environnementales au sein d'une science environnementale unique. De plus, subsistait la question de savoir si une telle superdiscipline était susceptible d'apporter une plus grande pertinence scientifique ou sociétale, qui favoriserait sa capacité explicative ou lui permettrait de mieux résoudre les problèmes. Abandonnant l'objectif de l'interdisciplinarité conceptuelle ou substantielle souhaitée au départ, on en venait à une forme d'interdisciplinarité plus instrumentale et même " partielle», puisqu'il s'agissait là des principaux niveaux d'interdisciplinarité effectivement obtenus, et sans doute les plus atteignables, tant dans le domaine de l'enseignement que dans celui de la recherche environnementale.

L'interdisciplinarité définie comme le développement d'une science environnementale unique ne semblait donc pas constituer un objectif atteignable. Dans le même temps, le modèle hiérarchique des principes directeurs et des disciplines fournissant leurs connaissances n'était pas praticable. Au contraire, l'interdisciplinarité ne semblait réalisable que par le truchement d'une longue collaboration entre les différentes disciplines, se guidant et s'alimentant mutuellement dans le cadre d'une interaction non hiérarchique. Quelles disciplines devaient être impliquées, et laquelle devait guider l'autre ou lui fournir des connaissances : telles paraissaient être les bonnes questions; des questions pour le coup purement pratiques, dépendant des compétences recherchées et des opportunités disponibles pour l'analyse et la résolution du problème traité. 


\section{Durabilité et interdisciplinarité}

Le bref exposé qui précède illustre l'importance revêtue par la question de l'interdisciplinarité dans le développement des sciences environnementales néerlandaises. Il montre bien en même temps que l'argumentation associée à cette question de l'interdisciplinarité a toujours reflété le statut des sciences environnementales du point de vue organisationnel : les arguments normatifs, qui caractérisaient l'idéologie antimoderniste de l'étape de formation, ont été écartés à mesure que les sciences environnementales se voyaient reconnues et s'institutionnalisaient. De même, l'objectif d'une science supradisciplinaire unique a semblé non seulement inatteignable, mais encore parfaitement superflu lorsque les sciences environnementales se furent progressivement établies et professionnalisées : l'interdisciplinarité est ainsi devenue une fructueuse et pragmatique collaboration entre disciplines.

La question de l'interdisciplinarité n'a probablement pas été aussi dominante dans les autres pays. Pourtant le cas néerlandais nous semble absolument représentatif. Depuis l'émergence des préoccupations environnementales dans les années 1970, des débats semblables ont eu lieu un peu partout dans le monde, comme en témoigne le grand nombre de publications, de conférences etc., consacrées au sujet dans cette période. La question a encore bénéficié d'un regain d'attention et s'est même trouvée mondialisée lorsque le concept de développement durable est apparu à la fin des années 1980, devenant le mot d'ordre de presque toutes les discussions sur l'environnement. Ce n'est pas ici le lieu d'entrer dans des considérations sur les présupposés de ce concept, sur l'attirance qu'il exerce et l'émotion qu'il suscite ou sur sa séduisante et trompeuse simplicité. On s'en tiendra à ses incidences sur la question de l'interdisciplinarité.

Dans sa réflexion sur l'interdisciplinarité vue comme une conséquence particulière du concept de durabilité, Sloep (1994) établit une distinction entre pluridisciplinarité et interdisciplinarité, qu'il considère comme les deux extrêmes d'un continuum. À l'un des extrêmes, la pluridisciplinarité se réfère à un ensemble de disciplines associées, mais multiples, sans objectifs explicatifs, méthodes ou théories en commun, utilisant une terminologie différente, ne définissant pas les problèmes de la même façon. À l'autre extrême, l'interdisciplinarité se caractérise par la volonté de résoudre un problème unique sur lequel toute l'attention est focalisée, à l'aide de théories explicatives homogènes et en utilisant un vocabulaire spécifique. Pour Sloep, le concept de durabilité marque un tournant en ce qu'il prolonge substantiellement l'interdisciplinarité en l'intégrant dans un cadre de référence, en l'ancrant dans un vocabulaire commun, suscitant le développement de théories et de paradigmes communs.
Bien que Sloep ait raison de souligner l'incidence que le concept de développement durable a eue sur les programmes d'enseignement et de recherche partout dans le monde, avec notamment l'apparition d'un vocabulaire commun permettant de traiter des questions liées à l'environnement, il nous semble, à dire vrai, trop optimiste quant au changement opéré vis-à-vis de l'interdisciplinarité. Et ceci principalement pour deux raisons. La première est qu'il considère la question avant tout comme un problème intellectuel et pense en termes de concepts et de théories communes, mais néglige plus ou moins les aspects organisationnels. Or, une des leçons que l'on peut tirer de ces trente années de débats sur l'interdisciplinarité est sans doute que, même s'il ne faut bien sûr pas sous-estimer l'importance des problèmes épistémologiques, théoriques et méthodologiques cruciaux auxquels se heurte l'interdisciplinarité, le développement de celle-ci est par-dessus tout affaire d'organisation : c'est (aussi) une question de gestion et il faut la traiter comme telle. Ce point sera examiné dans les deuxième et troisième parties. En second lieu, Sloep semble trop optimiste lorsqu'il pense assister, de son propre aveu un peu prématurément, à la mise en place d'une théorie du développement durable. Quoi qu'il en soit, il a tout à fait raison de mettre en avant la capacité du concept de développement durable à susciter une coopération interdisciplinaire accrue. Depuis qu'il a été admis comme fil conducteur de nombreux programmes de recherche environnementale, tant au plan national qu'international, il a du moins créé une atmosphère de préoccupation commune, tout en fournissant des concepts communs.

Blowers et Glasbergen (1995), pour leur part, suggèrent que le concept de durabilité prolonge de lui-même l'interdisciplinarité, dans la mesure où il plaide pour une coopération accrue entre les sciences de la nature et les sciences sociales. Selon eux, la «durabilité » est à l'origine un concept scientifique qui se réfère à l'idée de la persistance de systèmes naturels dans le temps et aux conditions physiques, biologiques ou autres, requises à cet effet. D'où les tentatives, principalement par des chercheurs en sciences de la nature et par certaines organisations de mouvements écologistes, visant à mettre en œuvre le concept de durabilité en élaborant des indicateurs évaluant, entre autres, la quantité d'énergie, les ressources non renouvelables, les terres etc., qu'un pays, une région ou même un individu donné peut se permettre d'exploiter de façon durable. En d'autres termes, on cherche à définir les conditions limites auxquelles une personne, une région ou un pays devraient s'en tenir. D'où les tentatives de traduire ces indicateurs de durabilité et ces conditions limites en termes d'objectifs pour les politiques environnementales. Par conséquent, la durabilité pourrait en soi servir aux chercheurs en sciences de la nature de principal critère d'évaluation de 
la viabilité environnementale de certaines activités sociétales ou économiques.

Le concept en cause, pourtant, n'est pas celui de durabilité mais celui de «développement durable». Le "développement » est un concept des sciences sociales, qui se réfère aux progrès des systèmes humains, pour reprendre Blowers et Glasbergen. Il se distingue de concepts proches et tout particulièrement de celui de «modernisation », concept très en vogue dans les sciences sociales il y a plus d'un siècle; et ce, pour deux raisons. Tout d'abord, contrairement au terme de modernisation, le concept de développement n'implique pas qu'il n'y ait qu'une seule voie valable vers une " modernité » unique (Becker et al., 1997). Cela ouvre des perspectives en matière d'apprentissage social, de réflexivité et d'autres concepts récents des sciences sociales, se référant à l'autoconfrontation et à l'autoréflexion, à l'examen critique et au changement social (Lash et al., 1996). En second lieu, alors que la rhétorique de la modernisation considérait l'homme et la société comme pleinement émancipés des contraintes biophysiques et des autres contraintes environnementales qui pesaient sur eux, le terme de développement, à coup sûr en tout cas lorsqu'il se trouve associé avec l'adjectif «durable», prend en compte l'environnement biophysique.

C'est pourquoi la «durabilité » semble porter sur la limite des activités sociales et s'inspirer principalement des résultats des recherches menées en sciences de la nature sur les "capacités de charge", les "niveaux sans effet » et autres normes faisant l'objet d'une évaluation graduelle allant de «faible » à «fort » selon la capacité de renouvellement du capital naturel. Le « développement durable », en revanche, se rapporte au comportement humain, aux capacités d'apprentissage, aux changements qui surviennent dans le métabolisme économique, aux opportunités politiques, etc. Ainsi, le développement durable, considéré comme processus de changement social dans un champ environnemental visiblement délimité mais encore inconnu, en appelle à l'implication de presque toutes les disciplines scientifiques au sein d'une coopération interdisciplinaire.

\section{Les avancées et les limites du débat sur l'interdisciplinarité}

Puisqu'apparemment la question de l'interdisciplinarité a été traitée, de façon détaillée ou incidente, depuis maintenant près de trente ans, et dans la mesure où le concept de durabilité a apporté un souffle nouveau et suscité une nouvelle exigence d'interdisciplinarité, quelles sont les leçons à tirer de cette expérience? Quelles sont les avancées produites par le débat sur l'interdisciplinarité ? C'est ce que l'on verra dans un premier temps. Mais aussi : quelles en sont les limites? Quelles incertitudes demeurent ? C'est ce que nous verrons ensuite. En un mot : où en sommes-nous?

\section{Avancées}

Le premier, et peut-être le plus important des bénéfices retirés de ce débat, est le fait que l'interdisciplinarité semble acceptée de façon générale comme attitude scientifique de base et comme présupposé méthodologique à l'analyse et à la résolution des problèmes environnementaux. Il est pourtant vrai que ce constat n'est souvent qu'apparent, puisque les disciplines traditionnelles continuent d'être les acteurs prédominants au sein de la communauté scientifique, en termes de comités de revues, de perspectives de carrière et de publications, comme en termes d'organisations professionnelles, d'accès aux financements, etc. Il serait intéressant d'entamer un projet de recherche comparative sur la manière dont on conçoit et dont on organise l'interdisciplinarité dans les sciences environnementales dans les pays européens et ailleurs, et de se demander dans quelle mesure cet état de fait peut s'expliquer par les traditions disciplinaires et les contextes institutionnels de chaque pays, comme Broekhans (2003) l'a fait pour les Pays-Bas. Les efforts de Hollaender (2004) sur ce point méritent d'être suivis. Dans son panorama des exemples allemands, suisses et autrichiens, elle se penche sur l'organisation interne de l'interdisciplinarité, mais prête moins d'attention au contexte institutionnel. Son approche pourrait néanmoins aisément se voir élargie, à la fois conceptuellement et géographiquement.

S'il est vrai que jusqu'à présent l'interdisciplinarité n'est pas encore suffisamment traduite sur le plan organisationnel, il est cependant indubitable qu'un changement a eu lieu en ce qui concerne le jugement de valeur porté sur elle. Ce changement d'attitude est en lui-même l'une des conditions qui rendront possible une approche globale des problèmes environnementaux dans l'enseignement, la recherche et la politique. Malgré la situation de pouvoir détenue par certaines disciplines traditionnelles et leur domination actuelle dans certains domaines environnementaux (ingénierie, météorologie, économie), ce changement d'attitude empêche qu'une discipline ou une communauté scientifique unique puisse prétendre au monopole de la compétence environnementale, et que les organismes gouvernementaux puissent concevoir des programmes monodisciplinaires de recherche environnementale. Cela constitue un changement majeur par rapport au début des années 1970, lorsque les ingénieurs, les chimistes et les médecins dominaient largement la discussion sur l'environnement, et que les chercheurs en sciences sociales devaient se battre pour prendre part au débat. On peut donc en déduire que la question environnementale et le débat sur l'interdisciplinarité qui lui 
est lié ont poussé les disciplines traditionnelles à redéfinir leur position sur ces questions, même si elles l'ont parfois fait pour des raisons prosaïques, comme des opportunités de financement, ou par volonté de se montrer politiquement correctes, comme Zanoni et al. (1997) le suggèrent avec raison.

La seconde avancée que le débat sur l'interdisciplinarité a produite est sans doute que les différentes conceptions de l'interdisciplinarité, les différents problèmes qu'elle pose et les différentes manières possibles de l'organiser ont été quelque peu clarifiés. Même si elle est définie de façon vague comme l'intégration des connaissances de différentes disciplines, l'interdisciplinarité suscite des questions telles que : l'intégration de quoi ? Et : des connaissances sur quoi? Ces questions relient d'ailleurs des questions relatives à l'intégration interdisciplinaire à des questions concernant l'interface entre sciences et politique, et aux débats, encore plus larges, sur les modes de production, de distribution et de validation du savoir, de la connaissance etc. (Nowotny et al., 2001 et autres). Ces considérations sont intimement liées entre elles, mais ce n'est pas le lieu ici d'entrer dans les détails. Au total, trois leçons principales peuvent être tirées de ces discussions.

En premier lieu, nous avons appris à ne pas souhaiter l'intégration ou la méta-intégration de théories, ni à vouloir forger une nouvelle discipline supradisciplinaire, car ces deux objectifs dépassent les perspectives actuelles (Dumas, 1996). Apprendre à utiliser des concepts communs, à discuter sur la base d'interprétations communes et à créer des modèles ou des langages communs, tel est l'objectif qui paraît le plus atteignable et constitue dans le même temps une tâche suffisamment complexe.

Nous avons également appris qu'une interdisciplinarité orientée vers la poursuite d'un objectif précis implique généralement, en réalité, la domination d'une discipline ou d'un ensemble de disciplines sur les autres, puisque la discipline principale entend imposer sa définition tant du problème que de l'objectif à atteindre, instrumentalisant les autres disciplines comme auxiliaires (Becker, 1997). Une recherche orientée vers un but donné tend à s'associer un ensemble de disciplines ordonnancé de façon hiérarchique, dont la plupart ne font que fournir leur savoir et leur compétence à la discipline située tout en haut de la pyramide ainsi constituée. C'est pourquoi des programmes de recherche orientés vers un objectif donné, par exemple visant une définition unidimensionnelle de la durabilité (celle des sciences de la nature) se sont avérés avoir une mauvaise approche de l'interdisciplinarité, car ils créent presque inévitablement une relation de maître à esclave entre les disciplines. C'est là un point sur lequel on reviendra.

Nous avons également appris qu'une approche orientée vers la résolution d'un problème offre de bien meilleures chances d'interdisciplinarité si elle commence par une définition commune du problème concerné. Pour ce qui est des programmes d'enseignement, les cours thématiques (que ce soit sur les déchets, sur l'énergie, sur les études d'impact ou l'analyse du cycle de vie) se sont avérés des points de départ satisfaisants pour une formation à des sciences de l'environnement interdisciplinaires (Leroy, 1997; Leroy et al., 2001). En particulier, les études de cas (sur la pollution, les transports, la rénovation urbaine, etc.) se sont montrées très prometteuses (Scholz, 1997), dans la mesure où elles simplifient et en même temps représentent dans toute leur complexité les problèmes inclus dans une approche scientifique des problèmes environnementaux. L'approche orientée vers la résolution d'un problème apparaît être la plus prometteuse en matière de recherche également, la plupart des chercheurs en sont d'accord.

Outre le partage d'une situation commune et d'une même définition du problème, Zanoni et al. (1997) ajoutent une autre condition au succès : «la prise de conscience des limites explicatives de sa propre discipline [...] prise de conscience qui, pour être fructueuse $[\ldots]$, ne peut être imposée de l'extérieur ». Dans leur esquisse de ce qu'ils présentent comme un «troisième modèle », qu'ils appellent de façon un peu sophistiquée "transdisciplinarité autoréflexive», Becker et al. (1997) finissent par aboutir au même point lorsqu'ils constatent que «cela commence par la reconnaissance explicite de ce que les questions de durabilité dépassent les traditionnels sujets d'étude des disciplines respectives » et avec « la prise de conscience des limites conceptuelles et méthodologiques liées au point de vue de chaque discipline».

\section{Limites, équivoques et incertitudes : les problèmes qui demeurent}

Les avancées et les bénéfices retirés du débat sur l'interdisciplinarité trouvent bien entendu leur pendant dans les limites de ce débat et dans les équivoques et les incertitudes qui subsistent. En premier lieu, malgré le changement d'attitude signalé précédemment et le lancement de programmes de recherche interdisciplinaires aux niveaux national et international, il existe encore peu de projets de recherche dans lesquels différentes disciplines fassent jeu égal, comme nous l'avons indiqué plus haut pour les Pays-Bas (Leroy, 1995), comme Hollaender (2001, 2004) le montre pour l'Allemagne, la Suisse et l'Autriche, et comme Zanoni et al. (1997) l'affirment pour le niveau international. Pour la plupart des observateurs, les programmes, bien que réputés interdisciplinaires, (i) sont en grande partie dominés par les sciences de la nature et (ii) s'avèrent souvent finalement à la rigueur pluridisciplinaires, si ce n'est même carrément monodisciplinaires. Des dispositions spécifiques destinées à promouvoir 
l'interdisciplinarité n'interviennent que de manière occasionnelle.

En second lieu, les efforts considérables mis en œuvre au cours des dernières décennies pour développer de nouveaux concepts, modèles et méthodes ne permettent pas d'affirmer avec certitude que les sciences environnementales disposent ou disposeront effectivement jamais d'un «corps de connaissances » commun et interdisciplinaire, ou de toute autre fondation scientifique solide dépassant le caractère imprécis de concepts tels que celui de développement durable. Le débat sur l'interdisciplinarité est à l'origine de certaines modélisations et métaphores scientifiques fructueuses au sujet de l'interaction homme-environnement - allant des modèles «Forces motrices-Pression-État-Impact-Réponse » aux métaphores du métabolisme. L'interdisciplinarité est néanmoins avant tout une question de transfert et d'intégration de méthodes, plus que d'élaboration de théories d'envergure. On rejoint ici ce qui a été dit plus haut sur le caractère superflu des tentatives de construire dans l'immédiat des métathéories, même si celles-ci peuvent devenir, à terme, les produits dérivés des efforts interdisciplinaires menés par la recherche empirique. En effet, on ne peut déterminer précisément dans quelle mesure ce développement pourrait contribuer à la capacité des sciences environnementales à expliquer ou à résoudre les problèmes. Dans le même temps, les sciences de l'environnement restent fragilisées par l'absence de théories ou de corps de connaissances communes au sein d'une communauté scientifique qui considère, à tort ou à raison, que des théories sophistiquées et éprouvées et un solide fondement scientifique sont autant de critères de prestige justifiant la reconnaissance universitaire.

Cela dit, les sciences environnementales ne souhaitent sans doute pas devoir leur statut universitaire aux critères scientifiques traditionnels, mais plutôt à leur intérêt sociétal et politique. Ce statut dépendrait donc en grande partie des aléas subis par la question environnementale dans l'actualité sociétale et politique, ce qui, dans de nombreux pays pour ne pas dire dans tous, constitue un fondement risqué en termes de contenu comme de financement. Néanmoins, il est probable que nous assistions aujourd'hui, de façon générale - mais tout particulièrement dans les sciences environnementales -, à une évolution significative de la raison d'être des sciences et de leur fondement. Cette évolution consiste dans le passage d'une production de connaissances fiables et complexes servant d'apport au système politique, à la mise à disposition de méthodes permettant de faire face à l'incertitude (Jasanoff, 1990 ; Roqueplo, 1991; Wynne, 1992, Nowotny et al., 2001). Une fois de plus, la question de l'interdisciplinarité s'avère intimement liée au statut des sciences de l'environnement dans leur contexte sociétal et politique en général, et à l'interface entre sciences et politique en particulier.
La quête de l'interdisciplinarité et l'orientation prédominante des sciences environnementales vers la recherche de solutions sont à l'origine d'un troisième problème que nous avons abordé tout à l'heure : la répartition inégale et injustifiée des tâches entre sciences de la nature et sciences sociales. De nombreux programmes de recherche environnementale, qu'ils soient conçus par les gouvernements des différents pays ou par les organisations internationales, suggèrent qu'il incombe aux sciences de la nature de définir le problème et plus particulièrement l'objectif tant du projet de recherche que des politiques qui s'en réclameront, limitant ainsi le rôle des sciences sociales à l'élaboration de stratégies pertinentes pour la résolution des problèmes. Cette conception de la répartition du travail entre sciences sociales et sciences de la nature ne concerne pas exclusivement les sciences appliquées : elle figure également dans certains manuels traitant des sciences environnementales (voir La Rivière, 1991; Benton, 1994; Leroy, 1995 pour une analyse détaillée). Étant donné le déséquilibre qui existe entre sciences de la nature et sciences sociales pour l'ensemble des questions environnementales, cette perspective orientée vers des objectifs donnés instrumentalise les sciences sociales dont il fait des victimes et les réduit au rang de technique sociale interventionniste, tout en exagérant leurs capacités de pilotage et d'incitation au changement. Cette perspective ne rend donc aucune justice à l'autonomie et au rôle critique des sciences sociales en matière d'analyse et de compréhension de la société, pas plus qu'elle ne les met en avant. En outre, elle occulte la nécessité d'une approche socio-scientifique des problèmes environnementaux. La «problématisation des définitions des problèmes » des autres, notamment des instances dirigeantes et des sciences de la nature, n'en représente pas moins, à mon sens, une tâche fondamentale pour les sciences sociales de l'environnement (Hannigan, 1995 ; Irwin, 2001).

Pour conclure cette partie, on peut dire que le débat en cours a clairement établi que l'interdisciplinarité, bien que nécessaire pour une approche détaillée des questions environnementales et faisant aujourd'hui, à ce titre, l'objet d'un large consensus, constitue un problème intellectuel complexe du fait des épistémologies divergentes et des différentes théories, méthodes, etc., utilisées par les sciences de la nature et les sciences sociales. Deux leçons majeures peuvent ainsi être tirées de ce débat : (i) les problèmes de l'interdisciplinarité et les stratégies visant à l'organiser sont étroitement liés au positionnement de la communauté scientifique dans son contexte politique global, et plus particulièrement à la relation entre les sciences de l'environnement et les politiques environnementales ; (ii) par ailleurs, outre ses aspects intellectuels, l'interdisciplinarité constitue un problème de gestion et d'organisation et doit être traitée comme telle. Cet impératif conduit à tenter d'énumérer quelques-uns 
des principaux facteurs permettant la réussite de l'organisation de l'interdisciplinarité dans la recherche et dans l'éducation.

\section{À la recherche d'une organisation de l'interdisciplinarité}

Dans les développements qui précèdent, des facteurs essentiels de réussite concernant la recherche interdisciplinaire ont été évoqués. Il faut revenir sur certains d'entre eux et en introduire d'autres, en établissant une distinction entre le niveau de l'attitude, celui des connaissances et celui de l'organisation. Le premier niveau, celui de l'attitude adoptée, consiste en une prise de conscience et une reconnaissance des limites explicatives de chaque discipline, ainsi qu'en une capacité d'accepter de rechercher de nouvelles manières de formuler les problèmes entre disciplines (Zanoni et al., 1997). Pour qu'elle soit effective, cette attitude doit nécessairement s'accompagner d'une acquisition partagée de connaissances (second niveau) sur les disciplines associées : ceci passe par une présentation préliminaire des perspectives et des questions de recherche propres à chacune d'elles et de la manière dont elles ont utilisé leurs paradigmes traditionnels (ou, au contraire, élaboré de nouveaux paradigmes) dans l'analyse des problèmes environnementaux. Cette connaissance de la formulation et des approches classiques d'un problème par les autres disciplines que la sienne est une condition sine qua non pour l'identification commune des champs et des objets de recherche en vue d'une éventuelle coopération interdisciplinaire, puisqu'elle permet de savoir quels concepts relient entre eux les terminologies de chaque discipline.

Quant aux conditions requises par l'interdisciplinarité au niveau organisationnel, il est aisé d'en comprendre la nécessité si l'on veut bien considérer que l'intégration des connaissances qui est au cœur même de l'interdisciplinarité consiste, en termes de gestion, en un processus de coordination de ces connaissances. Ce processus se doit donc d'être organisé. D'après les théories de la gestion, la coordination d'activités diverses mais interdépendantes peut se voir réalisée par le biais de différentes stratégies : $1^{\prime}$ « adaptation réciproque», la «normalisation ou standardisation », la "structuration hiérarchique », la "départementalisation ou la division nette des tâches", l'utilisation d' « intégrateurs » ou de « coordinateurs » (indépendants), ou encore le concours de "comités» ou de "groupes de travail ad hoc», etc. Certaines de ces stratégies peuvent d'avance être écartées comme inadaptées à la recherche interdisciplinaire, car elles supposent une organisation établie, alors que la recherche interdisciplinaire se déroule souvent dans un cadre interorganisationnel plus ou moins expérimental, préorganisationnel et novateur, et dans des structures matricielles temporaires parfois même choisies volontairement. La normalisation, pour sa part, est en outre rarement utilisée pour coordonner entre eux des professionnels. À l'inverse, les autres stratégies semblent fréquemment utilisées, que ce soit de manière explicite ou inconsciente, comme en témoignent les travaux de Zanoni (1997) et de Hollaender (2001).

L'expérience montre que «l'adaptation réciproque » se heurte à de sérieuses difficultés pour se mettre en place entre les professionnels de la recherche; et ceci a fortiori s'ils viennent de disciplines différentes. À la lecture des lignes qui précèdent, on aura compris pourquoi : de longues discussions et négociations visant à comprendre les différentes formulations des problèmes et à confronter des points de vues divergents sur des concepts communs constituent le préalable nécessaire à l'approfondissement de l'interdisciplinarité. C'est pourquoi seuls des ateliers, des conférences, des réunions d'experts, etc., sont susceptibles de créer les conditions d'une pratique interdisciplinaire digne de ce nom. Il y a là une contrainte spécifique fondamentale de la recherche interdisciplinaire. Elle exige évidemment du temps et des moyens financiers propres. Il faut aussi que les participants soient rompus aux techniques de discussion et de négociation, qu'ils connaissent la dynamique de groupe et qu'ils soient en mesure d'utiliser des compétences et des outils de facilitation. Dans certains cas, la nomination d'un coordinateur ou d'un groupe de travail intégrateur a été couronnée de succès; dans d'autres cas, elle a entraîné une "départementalisation » rapide et infructueuse. De toute évidence, plus le projet ou le programme est conséquent et plus le besoin de ce type de groupe de travail se fait sentir ; toutefois, le risque d'assister à d'une « départementalisation» non souhaitée augmente d'autant et procure un alibi aux participants qui se déchargent de la responsabilité de l'intégration. Il n'est pas surprenant de constater que la coordination "par hiérarchie », qui est plutôt utile dans les projets propres à une discipline et assortis d'objectifs et de méthodes bien déterminés, semble également efficace lorsque les projets interdisciplinaires ont des objectifs, des stratégies et des méthodes clairement délimités et définis de manière adéquate - ce qui est rarement le cas. Comme on l'a vu plus haut, la conduite hiérarchique de projets interdisciplinaires est généralement associée à des projets orientés vers des objectifs donnés dans lesquels, le plus souvent, une discipline fixe l'objectif et place les autres en situation de fournisseurs de connaissances, instrumentalisés. Cette technique se rapproche de ce que les gestionnaires appellent une "coordination par normalisation des résultats ou des cibles ». Cette dernière semble porter ses fruits dans un certain nombre de cas, mais elle est contreproductive dans les autres.

Des lignes qui précèdent peuvent être tirées des conclusions concernant l'enseignement. Elles valent en 
effet pour les connaissances et les compétences qu'un étudiant participant à un projet interdisciplinaire est censé avoir. La manière dont les étudiants sont formés dans une perspective interdisciplinaire au sein de l'Université de Nimègue a déjà été décrite (Leroy, 1997; Leroy et al., 2001). Il est néanmoins utile de présenter ici, quoique très brièvement, deux concepts : celui d' ' attitude interdisciplinaire » et celui de «spécialiste en série ». Derrière ces deux expressions, on retrouve les prises de positions de principes déjà exposées dans la seconde partie de ce texte.

L'option en faveur d'une «attitude interdisciplinaire» découle tout d'abord de l'idée selon laquelle il est impossible d'arriver à une interdisciplinarité de contenu et à une science de l'environnement supradisciplinaire unique. L'objectif ne devrait donc pas être de tenter de former les étudiants pour en faire des chercheurs en environnement généralistes. Ce concept résulte par ailleurs de la conviction selon laquelle l'interdisciplinarité consiste en une attitude, plus qu'en une question d'intégration des connaissances de différentes disciplines. L'interdisciplinarité dans les sciences de l'environnement devrait se concevoir comme une action réciproque, pragmatique et critique de diverses disciplines, ce qui requiert une interaction entre les différentes disciplines en fonction du problème en cause, et une coopération dans le cadre d'apports et de soutiens mutuels. Cette attitude, l'on peut, dans une certaine mesure, la provoquer, la soutenir et l'enseigner. L'attitude interdisciplinaire apparaît ainsi à l'heure actuelle comme la perception la plus réaliste de l'objectif que pourraient et devraient poursuivre les programmes universitaires. Certains des éléments nécessaires pour forger cette attitude ont déjà été évoqués. Redisons en bref qu'il s'agit de connaître et de comprendre les formulations des problèmes propres aux différentes disciplines, d'être attentif à la recherche d'éléments convergents entre elles, et d'être familier des concepts et des techniques qui peuvent permettre de jeter des ponts entre elles.

Le concept de "spécialiste en série » est basé sur la même philosophie. Il concerne les étudiants qui disposent d'une bonne formation dans une ou deux disciplines spécifiques et qui, de ce fait, sont capables d'approfondir d'autres disciplines de manière successive et d'en apprécier aussi bien les points forts que les limites, tout en restant habitués à leurs approches particulières des problèmes environnementaux. Cette attitude et cette aptitude peuvent tout aussi bien être développées et faire l'objet de formations. L'association entre l'étude approfondie d'une discipline, d'une part, et une attitude interdisciplinaire, d'autre part, permet aux «spécialistes en série » de jouer un rôle particulier dans un cadre interdisciplinaire, étant donné qu'ils peuvent "modifier » de manière relativement aisée leur approche vis-à-vis d'une discipline donnée. Ces capacités font d'eux les animateurs et les intégrateurs par excellence de la recherche environnementale interdisciplinaire. C'est pourquoi ils sont susceptibles de répondre aux attentes de la question environnementale - et du marché du travail.

\section{Références}

Becker, E., Jahn, T., Stiess, I., Wehling, P., 1997. Sustainability: a Cross-Disciplinary Concept for Social Transformations, Frankfurt/Paris, Unesco.

Benton, T., 1994, Biology, social theory in the environmental debate, in Redclift, M., Benton, T. (Eds), Social Theory and the Global Environment, London/New York, Routledge, 28-50.

Blowers, A., Glasbergen, P., 1995. The search for sustainable development, in Glasbergen, P., Blowers, A. (Eds), Environmental Policy in an International Context, London, Arnold/Open University, book 2, 163-183.

Broekhans, B., 2003. Hoe milieukunde geschiedenis werd. Demarcatie van een maatschappelijk relevante wetenschap, 19702000 (Comment la science de l'environnement est devenue histoire. Démarcation d'une science à pertinence sociale, 1970-2000), Nijmegen, Nijmegen University Press.

De Groot, W.T., 1992. Environmental Science Theory, Amsterdam, Elsevier.

Dumas, B., 1996. Science normale et sciences humaines : les voies de l'interdisciplinarité pour la résolution des problèmes environnementaux, in Tessier, R., Vaillancourt, J.-G., La Recherche sociale en environnement, Montréal, Les Presses de l'Université de Montréal, 75-88.

Hannigan, J., 1995. Environmental Sociology, London/New York, Routledge.

Hollaender, K., 2001. Interdisziplinarität in den Umweltwissenschaften: Konzeption(en), Organisation, Erfahrungen, in Fränzle, O., Müller, F., und Schröder, W. (Eds), Handbuch der Umweltwissenschaften, Landsberg am Lech, Ecomed Verlag.

Hollaender, K., 2004. Interdisziplinäre Forschung: Merkmale, Einflussfaktoren und Effekte.Thèse de doctorat de l'Université de Cologne, online via http://kups.ub.uni-koeln.de

Irwin, A., 2001. Sociology and the Environment, Cambridge, Polity Press.

Jamison, A., 1996. The Shaping of the Global Environmental Agenda: The Role of Non-Governmental Organisations, in Lash, S., Szerszynski, B., Wynne, B. (Eds), Risk, Environment and Modernity. Towards a New Ecology, London, Sage, 224245.

Jasanoff, S., 1990. The Fifth Branch. Science Advisers as Policy Makers, Harvard, Harvard University Press.

Jórgensen, S.E., Johnsen, I., 1981. Principles of Environmental Science and Technology, Amsterdam, Elsevier.

La Rivière, J.M.W., 1991. Co-operation between natural and social sciences in global change research: imperatives, realities, opportunities, International Social Science Journal, 130, 619-626.

Lash, S., Szerszynski, B., Wynne, B. (Eds), 1996. Risk, Environment and Modernity. Towards a New Ecology, London, Sage.

Leroy, P., 1995. Environmental Science as a Vocation, Lecture inaugural, Nijmegen, Nijmegen University Press.

Leroy, P., 1997. Interdisciplinarity as a Serial Specialists' Attitude: Dutch Environmental Sciences and the Nijmegen Experience 
with Interdisciplinarity, paper communicated at the AUDES Conference on Interdisciplinarity in University Education, Chambéry, France, September 1997.

Leroy, P., Van den Bosch, H., Ligthart, S., 2001. The Role of Project-Based Learning in the "Political and Socials Sciences of the Environment" Curriculum at Nijmegen University, International Journal for Sustainability in Higher Education, 2, 1, 8-20.

Mishan, E., 1968. The Costs of Economic Growth, London, Pelican Books.

Nebel, B.J., Wright, R.T., 1993. Environmental Science, Englewood Cliffs, Prentice Hall.

Nowotny H., Scott P., Gibbons, M., 2001, Re-thinking Science. Knowledge and the Public in an Age of Uncertainty, Cambridge, Polity Press.

O'Riordan, T. (Ed.), 1995. Environmental Science for Environmental Management, Harlow, Longman.

Roqueplo, Ph., 1991. L'expertise scientifique : convergence ou conflit de rationalité?, in Theys, J., et al., Environnement, science et politique, Paris, Germes, 43-80.

Roszak, Th., 1972. Where the Wasteland Ends, Doubleday, Garden City Publishers.

Reçu le 10 mars 2004. Accepté le 3 juin 2004.
Scholz, R., Stünzl, J., Mieg, H.A., Bösch, S., 1997. Methods for Interface Management: Interdisciplinary ETH-UNS Case Studies for Sustainability, paper presented at the Conference "Science for a Sustainable Society: Integrating Natural and Social Sciences", Roskilde (Denmark), October 1997.

Sloep, P.B., 1994. The Impact of "Sustainability" on the Field of Environmental Science, in Skirbekk, G. (Ed.), The Notion of Sustainability and its Normative Implications, Oslo, Scandinavian University Press, 29-55.

Udo de Haes, H.A., 1984. Milieukunde, begripsbepaling en afbakening (Environmental Science: Concepts and Boundaries), in Boersema, J.J., et al. (Ed.), Basisboek Milieukunde (Environmental Science Textbook), Meppel/Amsterdam, Boom, 17-30 (en néerlandais).

Wynne, B., 1992. Uncertainty and Environmental Learning, Global Environmental Change, 2, 111-127.

Zanoni, M., Vargas, M., Pivot, A., Raynaut, C., Lescure, J.-P., Quensière, J., 1997. La Recherche en environnement. À propos de quelques pratiques interdisciplinaires, unpublished paper resulting from an international workshop on interdisciplinary scientific practices (University of Campinas, Brasil, December 1996).

To access this journal online: www.edpsciences.org 